\title{
Synthesis and study of antimicrobial activity of new tetralone esters
}

\author{
Krishna Mudeenahally Hucchegowda, Basavaraju Yeriyur Basavaiah *, \\ Umesha Basavaiah and Shivakumar Santhekasalagere Basavaiah
}

Department of Studies in Chemistry, University of Mysore, Manasagangotri, Mysore-570 006, Karnataka, India

*Corresponding author at: Department of Studies in Chemistry, University of Mysore, Manasagangotri, Mysore-570 006, Karnataka, India. Tel.: +91.948.0441804. Fax: +91.821.2421263. E-mail address: basavaraju yb@yahoo.co.in (Y.B. Basavaraju).

\section{ARTICLE INFORMATION}

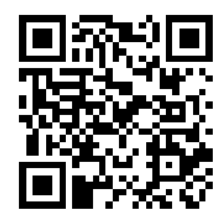

DOI: 10.5155 /eurjchem.5.4.584-587.1093

Received: 17 May 2014

Received in revised form: 28 June 2014

Accepted: 01 July 2014

Online: 31 December 2014

\section{KEYWORDS}

Tetralone esters

Cyclopropanation

Antimicrobial activity

Friedel-Crafts acylation

Claisen-Schmidt reaction

Ethyl monochloro acetate

\section{Introduction}

Podophyllotoxin (1) is a strong antimitotic agent [1,2]. Podophyllin is a resinous extract of two important medicinal plants Podophyllum emodi and Podophyllum peltatum belonging to the family of Berberidaceae [3-6]. It has also been extracted from many other plants of podophyllum species. Podophyllotoxin and its derivatives also exhibit strong antiviral and neoplastic activity [7]. The use of podophyllotoxin in cancer chemotherapy is restricted due to its toxic side effects and unfavourable solubility.

The semi-synthetic derivatives of podophyllotoxin, etoposide (VP-16, 2), teniposide (VM-26, 3) are used in the treatment of cancers, including small-cell lung cancer, lymphoma, testicular carcinoma and Kaposi's sarcoma (Figure 1) $[8,9]$. Some of its synthetic derivatives exhibit cathartic, cytotoxic and anticancer activities [10-12]. In view of these reports, it was decided to synthesize new tetralone esters (9ad) to study their structure activity relationship by modifying structures of podophyllotoxin.

\section{Experimental}

\subsection{Materials and methods}

All reagents and chemicals were purchased from Merck chemicals and were used without further purification. Melting points were determined by open capillary method and are uncorrected. The IR spectra were recorded on a FT-IR in $\mathrm{KBr}$ disc or Nujol. The ${ }^{1} \mathrm{H}$ NMR spectra were recorded on Jeol $300 \mathrm{MHz}$ and Jeol GSX-400 spectrometer using $\mathrm{CDCl}_{3}$ or DMSO$d_{6}$ as solvent and TMS as an internal reference. ${ }^{13} \mathrm{C}$ NMR $(100$ $\mathrm{MHz}$ ) spectra were recorded on Bruker DRX-400 instrument with DMSO- $d_{6}$ solvent. The chemical shifts were expressed in $\delta$ ppm values. The mass spectra (ESI-MS) were recorded by Bruker daltonics on ESQUIRE-3000 instrument.

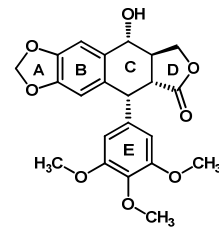

1

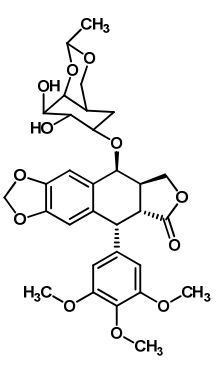

2

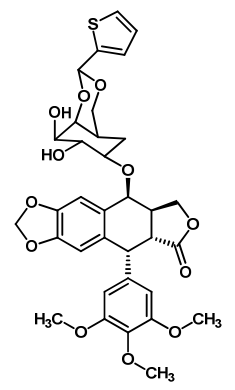

3
Figure 1. The structure of podophyllotoxin (1) and its semi synthetic derivatives etoposide (2) and teniposide (3). 

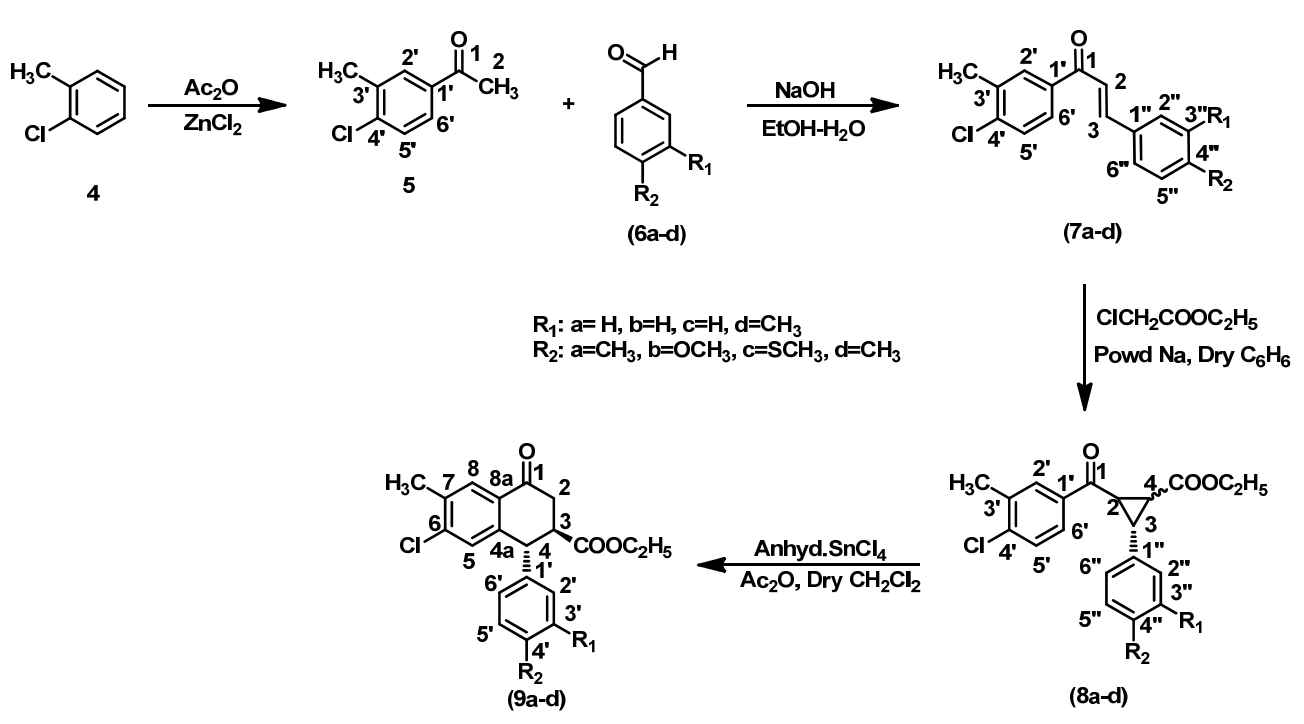

Scheme 1

The purity of the compounds was checked by TLC on silica gel glass plates in benzene:ethyl acetate mixture $(7: 0.5, v: v)$. The compounds were purified by column chromatography using silica gel (60-120 mesh) as adsorbent and benzene as eluent.

\subsection{Synthesis}

\subsubsection{Synthesis of acetophenone (5)}

2-Chloro toluene (4) (10 g, $0.079 \mathrm{~mol})$ in acetic anhydride $(50 \mathrm{~mL})$ containing fused zinc chloride $(10.76 \mathrm{~g}, 0.079 \mathrm{~mol})$ were stirred at room temperature for $12 \mathrm{~h}$. After usual workup, the product was obtained and it was recrystallized from ethanol (Scheme 1).

4'-Chloro-3'-methyl-acetophenone (5): Color: Colourless liquid. Yield: $93.81 \%$. B.p.: $104-105^{\circ} \mathrm{C}$. IR $\left(\mathrm{KBr}, v, \mathrm{~cm}^{-1}\right): 1673$ $(\mathrm{C}=0), 1598$ (C=C). ${ }^{1} \mathrm{H}$ NMR $\left(400 \mathrm{MHz}, \mathrm{CDCl}_{3}, \delta, \mathrm{ppm}\right): 2.32$ (s, $\left.3 \mathrm{H}, \mathrm{CH}_{3}\right), 2.49-2.54\left(\mathrm{~s}, 3 \mathrm{H}, \mathrm{COCH}_{3}\right), 7.34-7.73(\mathrm{~m}, 3 \mathrm{H}, \mathrm{Ar}-\mathrm{H}) .{ }^{13} \mathrm{C}$ NMR (100 MHz, DMSO- $\left.d_{6}, \delta, \mathrm{ppm}\right): 199.4$ (C-1), 138.6 (C-4'), 135.5 (C-3'), 134.7 (C-1'), 130.3 (C-2'), 127.9 (C-5'), 126.8 (C6'), 28.8 (C-2), $14.9\left(3^{\prime}-\mathrm{CH}_{3}\right)$. MS (ESI, $\left.m / z\right): 168.03\left(\mathrm{M}^{+}\right)$. Anal. calcd. for $\mathrm{C}_{9} \mathrm{H}_{9} \mathrm{ClO}: \mathrm{C}, 64.11 ; \mathrm{H}, 5.38$. Found: $\mathrm{C}, 64.08 ; \mathrm{H}, 5.35 \%$.

\subsubsection{General procedure for the synthesis of chalcones (7a- d)}

4'-Chloro-3'-methyl-acetophenone (5) (5 g, $0.0296 \mathrm{~mol})$ and substituted benzaldehydes (6a-d) $(0.0296 \mathrm{~mol})$ were stirred vigorously in water $(40 \mathrm{~mL})$ and ethanol $(25 \mathrm{~mL})$ mixture in the presence of sodium hydroxide $(1.18 \mathrm{~g}, 0.0296$ mol) at $15-30{ }^{\circ} \mathrm{C}$ for $4 \mathrm{~h}$. The reaction mixture was kept overnight in an ice bath. The precipitated products were filtered and recrystallized from ethanol (Scheme 1).

1-(4'-Chloro-3'-methyl-phenyl)-3-(4"'-methylphenyl)-prop-2ene-1-one (7a): Color: Pale yellow solid. Yield: 98.6\%. M.p.: 96$98{ }^{\circ} \mathrm{C}$. IR ( $\left.\mathrm{KBr}, v, \mathrm{~cm}^{-1}\right): 1665(\mathrm{C}=0), 1594(\mathrm{C}=\mathrm{C}) .{ }^{1} \mathrm{H}$ NMR $(400$ $\mathrm{MHz}, \mathrm{CDCl}_{3}, \delta, \mathrm{ppm}$ ): 2.32 (s, 3H, $\mathrm{CH}_{3}$ ), 2.36 (s, 3H, $\mathrm{CH}_{3}$ ), 7.04$7.62(\mathrm{~m}, 8 \mathrm{H}, \mathrm{Ar}-\mathrm{H}, \alpha-\mathrm{CH}), 8.02(\mathrm{~d}, 1 \mathrm{H}, J=12 \mathrm{~Hz}, \beta-\mathrm{CH}) .{ }^{13} \mathrm{C}$ NMR (100 MHz, DMSO- $d_{6}, \delta, \mathrm{ppm}$ ): 188.9 (C-1), 144.7 (C-3), 139.5 (C4'), 137.1 (C-4"), 136.3 (C-3'), 135.2 (C-1'), 131.8 (C-1"), 130.7 (C-2'), 128.9 (C-5'), 128.1 (C-3", C-5"), 127.9 (C-6'), 126.1 (C-2",

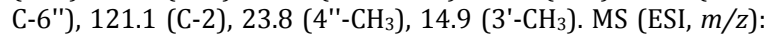
$270.08\left(\mathrm{M}^{+}\right)$. Anal. calcd. for $\mathrm{C}_{17} \mathrm{H}_{15} \mathrm{ClO}$ : C, 75.41; H, 5.58. Found: C, 75.38; H, 5.54\%.
1-(4'-Chloro-3'-methyl-phenyl)-3-(4'--methoxyphenyl)-prop2-ene-1-one (7b): Color: Yellow solid. Yield: 92.3\%. M.p.: 102$104{ }^{\circ} \mathrm{C}$. IR ( $\left.\mathrm{KBr}, v, \mathrm{~cm}^{-1}\right): 1662(\mathrm{C}=0), 1591(\mathrm{C}=\mathrm{C}) .{ }^{1} \mathrm{H}$ NMR $(400$ MHz, DMSO, $\delta, \mathrm{ppm}): 2.31\left(\mathrm{~s}, 3 \mathrm{H}, \mathrm{CH}_{3}\right), 3.82\left(\mathrm{~s}, 3 \mathrm{H}, \mathrm{OCH}_{3}\right), 6.84-$ $7.78(\mathrm{~m}, 8 \mathrm{H}, \mathrm{Ar}-\mathrm{H}, \alpha-\mathrm{CH}), 8.05(\mathrm{~d}, 1 \mathrm{H}, J=13 \mathrm{~Hz}, \beta-\mathrm{CH}) .{ }^{13} \mathrm{C}$ NMR (100 MHz, DMSO- $d_{6}, \delta, \mathrm{ppm}$ ): 188.8 (C-1), 159.2 (C-4"), 145.1 (C-3), 139.9 (C-4'), 136.4 (C-3'), 135.4 (C-1'), 131.3 (C-2'), 129.1 (C-5'), 128.1 (C-6'), 127.2 (C-1"), 127 (C-2", C-6"), 121.3 (C-2), 114.1 (C-3", C-5"), $55.7\left(\mathrm{OCH}_{3}\right), 15.1\left(3^{\prime}-\mathrm{CH}_{3}\right)$. MS (ESI, $\left.\mathrm{m} / \mathrm{z}\right)$ : $286.08\left(\mathrm{M}^{+}\right)$. Anal. calcd. for $\mathrm{C}_{17} \mathrm{H}_{15} \mathrm{ClO}_{2}$ : C, 71.20; $\mathrm{H}, 5.27$. Found: C, 71.18; H, 5.24\%.

1-(4'-Chloro-3'-methyl-phenyl)-3-(4"-methylthiophenyl)prop-2-ene-1-one (7c): Color: Yellow solid. Yield: $91.6 \%$. M.p.: 109-111 ${ }^{\circ} \mathrm{C}$. IR (KBr, $\left.v, \mathrm{~cm}^{-1}\right): 1667$ (C=0), 1598 (C=C). ${ }^{1} \mathrm{H}$ NMR ( $\left.400 \mathrm{MHz}, \mathrm{CDCl}_{3}, \delta, \mathrm{ppm}\right): 2.33\left(\mathrm{~s}, 3 \mathrm{H}, \mathrm{CH}_{3}\right), 2.51\left(\mathrm{~s}, 3 \mathrm{H}, \mathrm{SCH}_{3}\right)$, 7.26-7.62 (m, 8H, Ar-H, $\alpha-\mathrm{CH}), 8.04(\mathrm{~d}, 1 \mathrm{H}, J=12 \mathrm{~Hz}, \beta-\mathrm{CH}) .{ }^{13} \mathrm{C}$ NMR (100 MHz, DMSO- $\left.d_{6}, \delta, \mathrm{ppm}\right): 189.1$ (C-1), 145.1 (C-3), 139.9 (C-4'), 136.2 (C-3'), 135.8 (C-1'), 135.1 (C-4'), 131.2 (C1", C-2'), 129.1 (C-5'), 128.1 (C-6'), 126.2 (C-3"', C-5'), 125.9 (C2", C-6"), 121.1 (C-2), 15.1 (3'- $\left.\mathrm{CH}_{3}\right), 14.8\left(\mathrm{SCH}_{3}\right)$. MS (ESI, $\left.m / z\right)$ : $302.05\left(\mathrm{M}^{+}\right)$. Anal. calcd. for $\mathrm{C}_{17} \mathrm{H}_{15} \mathrm{ClOS}$ : C, 67.43; H, 4.99 . Found: C, 67.41; H, 4.96\%.

1-(4'-Chloro-3'-methyl-phenyl)-3-(3",4' '-dimethylphenyl)prop-2-ene-1-one (7d): Color: Yellow solid. Yield: 94.7\%. M.p.: 121-123 ${ }^{\circ}$ C. IR (KBr, $\left.v, \mathrm{~cm}^{-1}\right)$ : 1665 (C=0), 1597 (C=C). ${ }^{1} \mathrm{H}$ NMR (400 MHz, $\left.\mathrm{CDCl}_{3}, \delta, \mathrm{ppm}\right): 2.34\left(\mathrm{~s}, 3 \mathrm{H}, 3^{\prime}-\mathrm{CH}_{3}\right), 2.34$ (s, 3H, 3"$\left.\mathrm{CH}_{3}\right), 2.34\left(\mathrm{~s}, 3 \mathrm{H}, 4\right.$ "'- $\left.^{-} \mathrm{CH}_{3}\right), 6.63-7.95(\mathrm{~m}, 7 \mathrm{H}, \mathrm{Ar}-\mathrm{H}, \alpha-\mathrm{CH}), 8.06$ $(\mathrm{d}, 1 \mathrm{H}, J=12 \mathrm{~Hz}, \beta-\mathrm{CH}) .{ }^{13} \mathrm{C}$ NMR $\left(100 \mathrm{MHz}, \mathrm{DMSO}-d_{6}, \delta, \mathrm{ppm}\right)$ : 189.3 (C-1), 144.8 (C-3), 140.1 (C-4'), 136.7 (C-3"), 136.1 (C-3'), 135.8 (C-4"), 135.2 (C-1'), 132.1 (C-1"), 131.5 (C-2'), 129.2 (C5'), 128.6 (C-5"), 128.1 (C-6'), 126.1 (C-2"), 123.1 (C-6"), 121.2 (C-2), $18.1\left(3^{\prime \prime}-\mathrm{CH}_{3}\right), 17.3\left(4 "-\mathrm{CH}_{3}\right), 14.9\left(3^{\prime}-\mathrm{CH}_{3}\right) . \mathrm{MS}$ (ESI, $\mathrm{m} / \mathrm{z}): 284.10\left(\mathrm{M}^{+}\right)$. Anal. calcd. for $\mathrm{C}_{18} \mathrm{H}_{17} \mathrm{ClO}: \mathrm{C}, 75.92 ; \mathrm{H}, 6.02$. Found: C, 75.89; H, 6.01\%.

\subsubsection{General procedure for the synthesis of cyclopropyl keto esters $(8 a-d)$}

Chalcones (7a-d) $(0.0184 \mathrm{~mol})$, freshly distilled ethyl monochloro acetate $(2.25 \mathrm{~g}, 0.0184 \mathrm{~mol})$ and powdered sodium $(0.8 \mathrm{~g}, 0.0368 \mathrm{~mol})$ were stirred in dry benzene $(120 \mathrm{~mL})$ at room temperature for $30 \mathrm{~h}$. The unreacted sodium and its salts were filtered off. The filtrate was washed with $5 \%$ aqueous sodium hydroxide solution $(2 \times 50 \mathrm{~mL}), 2 \%$ brine solution $(2 \times$ $50 \mathrm{~mL}$ ) and dried over anhydrous sodium sulphate. The solvent 
was removed by distillation to give a crude product, which was purified by column chromatography using chloroform as eluent. The products were recrystallized from ethanol (Scheme 1).

Ethyl-2-(4'-chloro-3'-methyl-benzoyl)-3-(4"-methylphenyl)cyclopropane-1-carboxylate (8a): Color: Brown solid. Yield: 86.3\%. M.p.: 113-115 ${ }^{\circ} \mathrm{C}$. IR (KBr, $v, \mathrm{~cm}^{-1}$ ): 1741 (COO), 1675 $(\mathrm{C}=0), 1597$ (C=C). ${ }^{1 \mathrm{H}} \mathrm{NMR}\left(400 \mathrm{MHz}, \mathrm{CDCl}_{3}, \delta, \mathrm{ppm}\right): 1.12-1.31$ (t, $\left.3 \mathrm{H}, J=4 \mathrm{~Hz}, \mathrm{COOCH}_{2} \mathrm{CH}_{3}\right), 1.94-2.78(\mathrm{~m}, 3 \mathrm{H}$, cyclopro- $\mathrm{CH})$, $2.31\left(\mathrm{~s}, 3 \mathrm{H}, 3\right.$ '- $\left.-\mathrm{CH}_{3}\right), 2.34\left(\mathrm{~s}, 3 \mathrm{H}, 4{ }^{\prime \prime}-\mathrm{CH}_{3}\right), 4.05-4.13(\mathrm{q}, 2 \mathrm{H}$, $\left.J=4 \mathrm{~Hz}, \mathrm{COOCH}_{2} \mathrm{CH}_{3}\right), 6.97-7.66(\mathrm{~m}, 7 \mathrm{H}, \mathrm{Ar}-\mathrm{H}) .{ }^{13} \mathrm{C}$ NMR $(100$ MHz, DMSO- $d_{6}, \delta, \mathrm{ppm}$ ): 192.3 (C-1), 170.8 (COO), 140.2 (C-1"), 138.6 (C-4'), 135.3 (C-3'), 134.4 (C-1', C-4'), 130.2 (C-2'), 128.6 (C-5'), 128.1 (C-3", C-5"), 127 (C-6'), 124.6 (C-2", C-6"), 61.5 $\left(\mathrm{COOCH}_{2} \mathrm{CH}_{3}\right), 35.9$ (C-3), 33.2 (C-4), $31.1(\mathrm{C}-2), 24.1\left(4 "-\mathrm{CH}_{3}\right)$, $15.1\left(3^{\prime \prime}-\mathrm{CH}_{3}\right), 13.8\left(\mathrm{COOCH}_{2} \mathrm{CH}_{3}\right)$. MS (ESI, $\left.m / z\right): 356.12\left(\mathrm{M}^{+}\right)$. Anal. calcd. for $\mathrm{C}_{21} \mathrm{H}_{21} \mathrm{ClO}_{3}$ : C, 70.68; $\mathrm{H}, 5.93$. Found: C, 70.66; $\mathrm{H}$, $5.91 \%$.

Ethyl-2-(4'-chloro-3'-methyl-benzoyl)-3-(4"-methoxyphenyl)cyclopropane-1-carboxylate (8b): Color: Brown solid. Yield: 82\%. M.p.: 103-105 ${ }^{\circ} \mathrm{C}$. IR (KBr, $\left.v, \mathrm{~cm}^{-1}\right): 1735$ (COO), 1674 $(\mathrm{C}=0), 1595(\mathrm{C}=\mathrm{C}) .{ }^{1} \mathrm{H}$ NMR $\left(400 \mathrm{MHz}, \mathrm{CDCl}_{3}, \delta, \mathrm{ppm}\right): 0.98-$ $1.29\left(\mathrm{t}, 3 \mathrm{H}, \mathrm{J}=4 \mathrm{~Hz}, \mathrm{COOCH}_{2} \mathrm{CH}_{3}\right), 1.95-2.75(\mathrm{~m}, 3 \mathrm{H}$, cyclopro- $\mathrm{CH})$, 2.35 (s, $\left.3 \mathrm{H}, \mathrm{CH}_{3}\right), 3.82\left(\mathrm{~s}, 3 \mathrm{H}, \mathrm{OCH}_{3}\right), 4.05-4.15(\mathrm{q}, 2 \mathrm{H}, J=4 \mathrm{~Hz}$, $\left.\mathrm{COOCH}_{2} \mathrm{CH}_{3}\right), 6.67-7.68(\mathrm{~m}, 7 \mathrm{H}, \mathrm{Ar}-\mathrm{H}) .{ }^{13} \mathrm{C}$ NMR $(100 \mathrm{MHz}$, DMSO- $d_{6}, \delta, \mathrm{ppm}$ ): 191.9 (C-1), 171.2 (COO), 156.8 (C-4"), 138.4 (C-4'), 135.2 (C-3'), 134.9 (C-1'), 134.2 (C-1'), 130.2 (C-2'), 128.3 (C-5'), 126.7 (C-6'), 125.8 (C-2", C-6"), 113.3 (C-3", C-5"), $61.6\left(\mathrm{COOCH}_{2} \mathrm{CH}_{3}\right), 55.3\left(\mathrm{OCH}_{3}\right), 36.1(\mathrm{C}-3), 33.2(\mathrm{C}-4), 29.9(\mathrm{C}-$ 2), $14.8\left(3^{\prime}-\mathrm{CH}_{3}\right), 13.9\left(\mathrm{COOCH}_{2} \mathrm{CH}_{3}\right)$. MS (ESI, $\left.m / z\right): 372.11$ $\left(\mathrm{M}^{+}\right)$. Anal. calcd. for $\mathrm{C}_{21} \mathrm{H}_{21} \mathrm{ClO}_{4}$ : C, 67.65; H, 5.68. Found: C, 67.61; H, 5.64\%.

Ethyl-2-(4'-chloro-3'-methyl-benzoyl)-3-(4"'-meththio phenyl)-cyclopropane-1-carboxylate (8c): Color: Brown solid. Yield: 84.6\%. M.p.: $120-122{ }^{\circ} \mathrm{C}$. IR (KBr, $\left.v, \mathrm{~cm}^{-1}\right): 1740$ (COO), $1678(\mathrm{C}=0), 1598$ (C=C). ${ }^{1} \mathrm{H}$ NMR (400 MHz, DMSO- $\left.d_{6}, \delta, \mathrm{ppm}\right)$ : 0.99-1.29 (t, $\left.3 \mathrm{H}, J=4 \mathrm{~Hz}, \mathrm{COOCH}_{2} \mathrm{CH}_{3}\right), 1.92-2.74(\mathrm{~m}, 3 \mathrm{H}$, cyclopro- $\mathrm{CH}), 2.34\left(\mathrm{~s}, 3 \mathrm{H}, \mathrm{CH}_{3}\right), 2.51\left(\mathrm{~s}, 3 \mathrm{H}, \mathrm{SCH}_{3}\right), 3.96-4.16$ (q, $\left.2 \mathrm{H}, J=4 \mathrm{~Hz}, \mathrm{COOCH}_{2} \mathrm{CH}_{3}\right), 7.01-7.66(\mathrm{~m}, 7 \mathrm{H}, \mathrm{Ar}-\mathrm{H}) .{ }^{13} \mathrm{C}$ NMR $(100$ MHz, DMSO- $\left.d_{6}, \delta, \mathrm{ppm}\right): 191.7$ (C-1), 170.9 (COO), 139.8 (C-1"), 138.2 (C-4'), 135.4 (C-3'), 134.2 (C-1'), 132.2 (C-4'), 130.1 (C2'), 128.4 (C-5'), 126.7 (C-6'), 126 (C-3", C-5"), 125 (C-2", C-6"), $61.4\left(\mathrm{COOCH}_{2} \mathrm{CH}_{3}\right), 36(\mathrm{C}-3), 33.1(\mathrm{C}-4), 30.8(\mathrm{C}-2), 15\left(3^{\prime}-\mathrm{CH}_{3}\right)$, $14.6\left(\mathrm{SCH}_{3}\right), 13.7\left(\mathrm{COOCH}_{2} \mathrm{CH}_{3}\right)$. MS (ESI, $\left.\mathrm{m} / \mathrm{z}\right): 388.09\left(\mathrm{M}^{+}\right)$. Anal. calcd. for $\mathrm{C}_{21} \mathrm{H}_{21} \mathrm{ClO}_{3} \mathrm{~S}$ : C, 64.85; H, 5.44. Found: C, 64.83; $\mathrm{H}, 5.41 \%$.

Ethyl-2-(4'-chloro-3'-methyl-benzoyl)-3-(3"',4"'-dimethyl phenyl)-cyclopropane-1-carboxylate (8d): Color: Brown solid. Yield: 87.3\%. M.p.: $130-132{ }^{\circ} \mathrm{C}$. IR ( $\left.\mathrm{KBr}, v, \mathrm{~cm}^{-1}\right): 1738$ (COO), $1677(\mathrm{C}=0), 1593(\mathrm{C}=\mathrm{C}) .{ }^{1} \mathrm{H}$ NMR $\left(400 \mathrm{MHz}, \mathrm{CDCl}_{3}, \delta, \mathrm{ppm}\right)$ : 0.98-1.30 (t, $\left.3 \mathrm{H}, J=4 \mathrm{~Hz}, \mathrm{COOCH}_{2} \mathrm{CH}_{3}\right), 1.92-2.73(\mathrm{~m}, 3 \mathrm{H}$, cyclopro-CH), $2.30\left(\mathrm{~s}, 3 \mathrm{H}, 3^{\prime}-\mathrm{CH}_{3}\right), 2.33\left(\mathrm{~s}, 3 \mathrm{H}, 3^{\prime \prime}-\mathrm{CH}_{3}\right), 2.36(\mathrm{~s}$, $\left.3 \mathrm{H}, 4 "-\mathrm{CH}_{3}\right), 4.07-4.12\left(\mathrm{q}, 2 \mathrm{H}, \mathrm{J}=4 \mathrm{~Hz}, \mathrm{COOCH}_{2} \mathrm{CH}_{3}\right), 6.81-7.64$ (m, 6H, Ar-H). ${ }^{13} \mathrm{C}$ NMR (100 MHz, DMSO- $\left.d_{6}, \delta, \mathrm{ppm}\right): 191.5$ (C1), 170.6 (COO), 139.5 (C-1'), 138.3 (C-4'), 135.8 (C-3"), 134.8 (C-3'), 134.4 (C-1'), 133.1 (C-4'), 129.8 (C-2'), 128.2 (C-5'), 127.9 (C-5"), 126.8 (C-6'), 126.1 (C-2"), 121.7 (C-6"), 61.2 $\left(\mathrm{COOCH}_{2} \mathrm{CH}_{3}\right), 36.2$ (C-3), 33.2 (C-4), 30.6 (C-2), 18.1 (3"- $\left.\mathrm{CH}_{3}\right)$, $17.7\left(4^{\prime \prime}-\mathrm{CH}_{3}\right), 14.6\left(3^{\prime}-\mathrm{CH}_{3}\right), 14\left(\mathrm{COOCH}_{2} \mathrm{CH}_{3}\right) . \mathrm{MS}(\mathrm{ESI}, \mathrm{m} / \mathrm{z})$ : $370.13\left(\mathrm{M}^{+}\right)$. Anal. calcd. for $\mathrm{C}_{22} \mathrm{H}_{23} \mathrm{ClO}_{3}$ : C, 71.25; $\mathrm{H}, 6.25$. Found: C, 71.23; H, 6.22\%.

\subsubsection{General procedure for the synthesis of tetralone esters $(9 a-d)$}

A solution of cyclopropyl keto esters (8a-d) $(0.0140 \mathrm{~mol})$ in dry dichloromethane $(75 \mathrm{~mL})$ was added dropwise to a magnetically stirred solution of anhydrous Stannic chloride $(3.65 \mathrm{~g}, 0.0140 \mathrm{~mol})$ and acetic anhydride $(2.86 \mathrm{~g}, 0.0280 \mathrm{~mol})$ in dichloromethane $(75 \mathrm{~mL})$ for half anone $\mathrm{h}$ at $0{ }^{\circ} \mathrm{C}$ and further stirred for $6 \mathrm{~h}$. After treating the reaction mixture with $5 \mathrm{~N} \mathrm{HCl}$ solution $(50 \mathrm{~mL})$, the organic layer was washed with $10 \%$ $\mathrm{NaOH}$ solution $(2 \times 50 \mathrm{~mL})$ and finally with water. The crude product was purified by column chromatography using benzene as eluent (Scheme 1).

3-Ethylcarboxy-4-(4'-methylphenyl)-6-chloro-7-methyl1-tetralone (9a): Color: Reddish brown semi solid. Yield: 87.3\%. IR (KBr, $v, \mathrm{~cm}^{-1}$ ): 1745 (COO), 1698 (C=0), 1592 (C=C). ${ }^{1} \mathrm{H}$ NMR (400 MHz, $\left.\mathrm{CDCl}_{3}, \delta, \mathrm{ppm}\right): 0.98-1.30(\mathrm{t}, 3 \mathrm{H}, J=4 \mathrm{~Hz}$, $\left.\mathrm{COOCH}_{2} \mathrm{CH}_{3}\right), 2.31\left(\mathrm{~s}, 3 \mathrm{H}, 7-\mathrm{CH}_{3}\right), 2.35\left(\mathrm{~s}, 3 \mathrm{H}, 4{ }^{\prime}-\mathrm{CH}_{3}\right), 2.76-3.02$ (dd, $\left.2 \mathrm{H}, \mathrm{CH}_{2}\right), 3.63(\mathrm{q}, 1 \mathrm{H}, J=4 \mathrm{~Hz}, \mathrm{CH}), 4.11-4.22(\mathrm{q}, 2 \mathrm{H}, J=4 \mathrm{~Hz}$, $\left.\mathrm{COOCH}_{2} \mathrm{CH}_{3}\right), 4.66(\mathrm{~d}, 1 \mathrm{H}, J=12 \mathrm{~Hz}, \mathrm{CH}), 7.15-7.58(\mathrm{~m}, 6 \mathrm{H}, \mathrm{Ar}-\mathrm{H})$. ${ }^{13} \mathrm{C}$ NMR (100 MHz, DMSO-d6, $\delta, \mathrm{ppm}$ ): 196.7 (C-1), 172.8 (C00), 139.9 (C-1'), 138.8 (C-6), 138.3 (C-4a), 135.4 (C-4'), 133.1 (C-7), 131.6 (C-8a), 130.7 (C-8), 129.2 (C-3', C-5'), 127.7 (C-2', C-6'), $127.6(\mathrm{C}-5), 61.5\left(\mathrm{COOCH}_{2} \mathrm{CH}_{3}\right), 45.1(\mathrm{C}-4), 40.8$ (C3), 37.2 (C-2), $24\left(4-\mathrm{CH}_{3}\right), 14.8\left(7-\mathrm{CH}_{3}\right), 13.9\left(\mathrm{COOCH}_{2} \mathrm{CH}_{3}\right)$. MS (ESI, $m / z$ ): $356.12\left(\mathrm{M}^{+}\right)$. Anal. calcd. for $\mathrm{C}_{21} \mathrm{H}_{21} \mathrm{ClO}_{3}: \mathrm{C}, 70.68 ; \mathrm{H}$, 5.93. Found: C, 70.65; H, 5.91\%.

3-Ethylcarboxy-4-(4'-methoxyphenyl)-6-chloro-7-methyl1-tetralone (9b): Color: Reddish brown semi solid. Yield: 82.6\%. IR (KBr, $\left.v, \mathrm{~cm}^{-1}\right)$ : 1742 (COO), $1696(\mathrm{C}=0), 1594(\mathrm{C}=\mathrm{C})$ ${ }^{1} \mathrm{H}$ NMR $\left(400 \mathrm{MHz}, \mathrm{CDCl}_{3}, \delta, \mathrm{ppm}\right): 1.28(\mathrm{t}, 3 \mathrm{H}, J=4 \mathrm{~Hz}$, $\left.\mathrm{COOCH}_{2} \mathrm{CH}_{3}\right), 2.33\left(\mathrm{~s}, 3 \mathrm{H}, \mathrm{CH}_{3}\right), 2.73-3.04\left(\mathrm{dd}, 2 \mathrm{H}, \mathrm{CH}_{2}\right), 3.62$ (q, $1 \mathrm{H}, J=3 \mathrm{~Hz}, \mathrm{CH}), 3.82\left(\mathrm{~s}, 3 \mathrm{H}, \mathrm{OCH}_{3}\right), 4.19(\mathrm{q}, 2 \mathrm{H}, J=4 \mathrm{~Hz}$, $\left.\mathrm{COOCH}_{2} \mathrm{CH}_{3}\right), 4.68(\mathrm{~d}, 1 \mathrm{H}, J=12 \mathrm{~Hz}, \mathrm{CH}), 6.92-7.57(\mathrm{~m}, 6 \mathrm{H}, \mathrm{Ar}-\mathrm{H})$. ${ }^{13} \mathrm{C}$ NMR (100 MHz, DMSO- $\left.d_{6}, \delta, \mathrm{ppm}\right): 196.3$ (C-1), 173.1 (CO0), 157.8 (C-4'), 139.1 (C-6), 138.6 (C-4a), 135.1 (C-1'), 132.9 (C-7), 131.9 (C-8a), 130.6 (C-8), 129 (C-2', C-6'), 127.2 (C5), $114.2\left(\mathrm{C}-3^{\prime}, \mathrm{C}^{5} 5^{\prime}\right), 61.3\left(\mathrm{COOCH}_{2} \mathrm{CH}_{3}\right), 55.4\left(\mathrm{OCH}_{3}\right), 45.2$ (C4), 40.6 (C-3), 37.2 (C-2), $15\left(7-\mathrm{CH}_{3}\right), 13.7\left(\mathrm{COOCH}_{2} \mathrm{CH}_{3}\right)$. MS (ESI, $m / z$ ): $372.11\left(\mathrm{M}^{+}\right)$. Anal. calcd. for $\mathrm{C}_{21} \mathrm{H}_{21} \mathrm{ClO}_{4}: \mathrm{C}, 67.65 ; \mathrm{H}$, 5.68. Found: C, 67.62; H, 5.66\%.

3-Ethylcarboxy-4-(4'-methylthiophenyl)-6-chloro-7-methyl1-tetralone (9c): Color: Reddish brown semi solid. Yield: $89.4 \%$. IR (KBr, $\left.v, \mathrm{~cm}^{-1}\right): 1748$ (COO), 1691 (C=0), $1583(\mathrm{C}=\mathrm{C}) .{ }^{1} \mathrm{H}$ NMR ( $\left.400 \mathrm{MHz}, \mathrm{CDCl}_{3}, \delta, \mathrm{ppm}\right): 1.28\left(\mathrm{t}, 3 \mathrm{H}, J=4 \mathrm{~Hz}, \mathrm{COOCH}_{2} \mathrm{CH}_{3}\right), 2.34$ (s, $\left.3 \mathrm{H}, \mathrm{CH}_{3}\right), 2.53$ (s, $3 \mathrm{H}, \mathrm{SCH}_{3}$ ), 2.74-3.03 (dd, $2 \mathrm{H}, \mathrm{CH}_{2}$ ), 3.60 (q, $1 \mathrm{H}, J=3 \mathrm{~Hz}, \mathrm{CH}), 4.21\left(\mathrm{q}, 2 \mathrm{H}, J=4 \mathrm{~Hz}, \mathrm{COOCH}_{2} \mathrm{CH}_{3}\right), 4.67(\mathrm{~d}, 1 \mathrm{H}$ $J=4 \mathrm{~Hz}, \mathrm{CH}), 7.13-7.56(\mathrm{~m}, 6 \mathrm{H}, \mathrm{Ar}-\mathrm{H}) .{ }^{13} \mathrm{C}$ NMR $(100 \mathrm{MHz}$, DMSO$\left.d_{6}, \delta, \mathrm{ppm}\right): 196.5$ (C-1), 172.3 (COO), 138.8 (C-4'), 139.1 (C-6), 138.2 (C-4a), 133.4 (C-4'), 133 (C-7), 131.4 (C-8a), 130.9 (C-8), 128.3 (C-2', C-6'), 127.4 (C-5), 127.1 (C-3', C-5'), 61.5 $\left(\mathrm{COOCH}_{2} \mathrm{CH}_{3}\right), 45.4(\mathrm{C}-4), 41.2(\mathrm{C}-3), 37.6(\mathrm{C}-2), 14.6\left(7-\mathrm{CH}_{3}\right)$ 13.9 $\left(\mathrm{SCH}_{3}\right), 13.2\left(\mathrm{COOCH}_{2} \mathrm{CH}_{3}\right)$. MS (ESI, $\left.\mathrm{m} / \mathrm{z}\right): 388.09\left(\mathrm{M}^{+}\right)$. Anal. calcd. for $\mathrm{C}_{21} \mathrm{H}_{21} \mathrm{ClO}_{3} \mathrm{~S}$ : C, 64.85; H, 5.44. Found: C, 64.83; $\mathrm{H}, 5.41 \%$.

3-Ethylcarboxy-4-(3',4'-dimethylphenyl)-6-chloro-7-methyl1-tetralone (9d): Color: Reddish brown semi solid. Yield: 79.8\%. IR (KBr, v, cm $\left.{ }^{-1}\right): 1744$ (COO), 1693 (C=0), 1599 (C=C). ${ }^{1} \mathrm{H}$ NMR $\left(400 \mathrm{MHz}, \mathrm{CDCl}_{3}, \delta, \mathrm{ppm}\right): 1.15-1.29(\mathrm{t}, 3 \mathrm{H}, J=4 \mathrm{~Hz}$, $\left.\mathrm{COOCH}_{2} \mathrm{CH}_{3}\right), 2.32\left(\mathrm{~s}, 3 \mathrm{H}, 7-\mathrm{CH}_{3}\right), 2.34\left(\mathrm{~s}, 3 \mathrm{H}, 3^{\prime}-\mathrm{CH}_{3}\right), 2.35(\mathrm{~s}$, $\left.3 \mathrm{H}, 4{ }^{\prime}-\mathrm{CH}_{3}\right), 2.71-3.02\left(\mathrm{dd}, 2 \mathrm{H}, \mathrm{CH}_{2}\right), 3.62(\mathrm{q}, 1 \mathrm{H}, J=3 \mathrm{~Hz}, \mathrm{CH})$, 4.18-4.22 (q, $\left.2 \mathrm{H}, J=4 \mathrm{~Hz}, \mathrm{COOCH}_{2} \mathrm{CH}_{3}\right), 4.68(\mathrm{~d}, 1 \mathrm{H}, J=4 \mathrm{~Hz}, \mathrm{CH})$, 6.97-7.57 (m, 5H, Ar-H). ${ }^{13} \mathrm{C}$ NMR (100 MHz, DMSO- $\left.d_{6}, \delta, p p m\right)$ : 195.7 (C-1), 172.1 (CO0), 139.3 (C-1'), 138.5 (C-6), 138 (C-4a), 137.1 (C-3'), 134.2 (C-4'), 133.1 (C-7), 131.6 (C-8a), 130.9 (C-8), 129.3 (C-2'), 128.6 (C-5'), 127.5 (C-5), 125 (C-6'), 61.1 $\left(\mathrm{COOCH}_{2} \mathrm{CH}_{3}\right), 45.8$ (C-4), 41 (C-3'), 37.4 (C-2), $18\left(3^{\prime}-\mathrm{CH}_{3}\right), 17.8$ $\left(4{ }^{\prime}-\mathrm{CH}_{3}\right), 15\left(7-\mathrm{CH}_{3}\right), 13.9\left(\mathrm{COOCH}_{2} \mathrm{CH}_{3}\right)$. MS (ESI, $\left.\mathrm{m} / \mathrm{z}\right): 370.13$ $\left(\mathrm{M}^{+}\right)$. Anal. calcd. forC $\mathrm{C}_{22} \mathrm{H}_{23} \mathrm{ClO}_{3}$ : C, 71.25; $\mathrm{H}, 6.25$. Found: C, 71.23; H, 6.24\%.

\subsection{Antimicrobial activity}

The newly synthesized compounds (9a-d) were evaluated for their in vitro antibacterial activity against the gram positive bacteria Staphylococcus aureus and the gram negative bacteria Escherichia coli by disc diffusion method [13]. The bioassay was carried out using Mueller-Hinton agar (Hi-Media) medium. Ciprofloxacin was used as a standard. Simillarly the in vitro antifungal activity was evaluated for the compounds 9a-d 
against Aspergillis niger and Candida albicans (recultured) by disc diffusion method [14] with Sabouraud's dextrose agar (HiMedia). Clotrimazole was used as a standard. Each compound was tested at a concentration of $100 \mu \mathrm{g} / \mathrm{mL}$ in DMSO for both activities. The plates were incubated at $35{ }^{\circ} \mathrm{C}$ for $24 \mathrm{~h}$ and the resulting zone of inhibition (in $\mathrm{mm}$ ) was measured.

\section{Results and discussion}

\subsection{Chemistry}

In this paper, the chalcone route has been followed with some changes in experimental procedure to synthesize new tetralone esters (9a-d) (Scheme 1) [15,16]. The 4'-chloro-3'methyl-acetophenone (5) was prepared in high yield by Friedel-Crafts acylation reaction of 2-chloro toluene (4) with acetic anhydride in the presence of fused zinc chloride [17]. The structure of acetophenone was confirmed by IR and ${ }^{1} \mathrm{H}$ NMR spectra. The IR spectra showed $C=C$ stretching frequency at $1598 \mathrm{~cm}^{-1}$ and $\mathrm{C}=0$ stretching frequency at $1673 \mathrm{~cm}^{-1}$. The ${ }^{1} \mathrm{H}$ NMR spectra signals corresponding to $\mathrm{COCH}_{3}$ appeared at 2.49$2.54 \mathrm{ppm}$.

The chalcones (7a-d) were prepared in excellent yields by Claisen-Schmidt reaction of 4'-chloro-3'-methyl-acetophenone (5) with benzaldehydes (6a-d) in the presence of sodium hydroxide in water-ethanol mixture [18]. The structures of chalcones were confirmed by IR and ${ }^{1} \mathrm{H}$ NMR spectra. The IR spectra of chalcones showed $\mathrm{C}=\mathrm{C}$ stretching frequency in the range of $1591-1598 \mathrm{~cm}^{-1}$ and $\mathrm{C}=0$ stretching frequency in the range of $1662-1667 \mathrm{~cm}^{-1}$. The ${ }^{1} \mathrm{H}$ NMR spectra signals corresponding to $\alpha-\mathrm{CH}$ and $\beta$ - $\mathrm{CH}$ of chalcones appeared at 7.62-7.95 ppm and 8.02-8.06 ppm with coupling constant $J=12 \mathrm{~Hz}$.

The cyclopropyl keto esters (8a-d) were prepared in good yields by the reaction of chalcones (7a-d) with ethyl monochloro acetate in the presence of powdered sodium in dry benzene [19]. The IR spectra showed stretching frequencies at 1593-1598, 1674-1678 and $1735-1741 \mathrm{~cm}^{-1}$ for $\mathrm{C}=\mathrm{C}, \mathrm{C}=\mathrm{O}$ and ester $\mathrm{C}=\mathrm{O}$ of cyclopropyl keto esters respectively. The ${ }^{1} \mathrm{H}$ NMR spectra signals of cyclopropyl $\mathrm{CH}$ protons appeared at $1.92-$ $2.78 \mathrm{ppm}$.

The tetralone esters (9a-d) were prepared in good yields by intramolecular cyclization of cyclopropyl keto esters (8a-d) in the presence of anhydrous stannic chloride and acetic anhydride in dry dichloromethane [20,21]. The structures of tetralone esters were based on IR, ${ }^{1} \mathrm{H}$ NMR, Mass spectra and elemental analysis data. The IR stretching frequencies of compounds (9a-d) showed at 1592-1599, 1691-1698 and $1742-1748 \mathrm{~cm}^{-1}$ for $\mathrm{C}=\mathrm{C}, \mathrm{C}=0$ and ester $\mathrm{C}=0$, respectively. The ${ }^{1} \mathrm{H}$ NMR spectra signals appeared at $0.98-1.30 \mathrm{ppm}$ and 4.11 $4.22 \mathrm{ppm}$ for $\mathrm{CH}_{2}$ and $\mathrm{CH}_{3}$ protons of tetralone esters with a coupling constant $J=4 \mathrm{~Hz}$.

\subsection{Antimicrobial activity}

\subsubsection{Antibacterial activity}

The synthesized compounds 9a-d were screened for their antibacterial activity. The compound 9a has low activity compared to standard (Ciprofloxacin) and compounds $\mathbf{9 b - d}$ against Escherichia colia nd Staphylococcus aureus at a concentration of $100 \mu \mathrm{g} / \mathrm{mL}$. Compound 9d has moderate activity and compounds $\mathbf{9 b}$ and $\mathbf{9 c}$ exhibited better activities compared to ciprofloxacin and compounds $9 \mathbf{a}$ and $9 \mathbf{d}$ at the same concentration. The bacterial zones of inhibition (in $\mathrm{mm}$ ) values were summarized in Table 1.

\subsubsection{Antifungal activity}

The synthesized compounds 9a-d were screened for their antifungal activity. Compounds $\mathbf{9 b}$ and $9 \mathbf{c}$ are more active when compared to standard (Clotrimazole). The compound 9a exhibited low activity and the compound 9d exhibited moderate activity against Aspergillis niger and Candida albicans at a concentration of $100 \mu \mathrm{g} / \mathrm{mL}$. The fungal zones of inhibition (in $\mathrm{mm}$ ) values were summarized in Table 2.

Table1. Antibacterial activity of the synthesized compounds 9a-d.

\begin{tabular}{lll}
\hline Compound & \multicolumn{2}{l}{ Zone of inhibition in (mm) } \\
\cline { 2 - 3 } & E. coli & S. aureus \\
\hline 9a & 9 & 11 \\
9b & 12 & 14 \\
9c & 27 & 28 \\
9d & 29 & 26 \\
\hline Ciprofloxacin & 21 & 23 \\
\hline
\end{tabular}

Table 2. Antifungal activity of the synthesized compounds 9a-d.

\begin{tabular}{lll}
\hline Compound & \multicolumn{2}{l}{ Zone of inhibition in ( $m$ m $)$} \\
\cline { 2 - 3 } & A. niger & C. albicans \\
\hline 9a & 8 & 10 \\
9b & 14 & 15 \\
9c & 23 & 24 \\
9d & 25 & 23 \\
\hline Clotrimazole & 22 & 20 \\
\hline
\end{tabular}

\section{Conclusion}

The new tetralone esters 9a-d were synthesized in good yields. They were screened for their antimicrobial activities. All the compounds exhibited better activities.

\section{Acknowledgement}

The authors are thankful to the director, Central Drug Research Institute (CDRI), Lucknow and Indian Institute of Technology (IIT), Chennai, for providing NMR, Mass spectra and elemental analysis data for our research compounds.

\section{References}

[1]. Smissman, E. E.; Murray, R. J.; McChesney, J. D. J. Med. Chem. 1976, 19, 148-153.

[2]. Jungi, W. F.; Senn, H. J. Cancer Chemother. Rep. 1975, 59, 737-742.

[3]. Podwyssotzki, V. Arch. Exp. Pathol. Pharmakol. 1880, 13, 29-52.

[4]. Jackson, D. E.; Dewick, P. M. Phytochemistry 1985, 24, 2407-2409.

[5]. Yalamati, D.; William, L. Curr. Med. Chem. 1998, 5, 205-252.

[6]. Kelly, M. G.; Hartwell, J. L. J. Natl. Cancer Inst. 1954, 14, 967-1010.

[7]. Canel, C.; Moraes, R. M.; Dayan, F. E.; Ferreira, D. Phytochemistry 2000, $54,115-120$.

[8]. Imbert, T. F. Biochimie 1998, 80(3), 207-222.

[9]. Gordaliza, M.; Garcia, P. A.; Miguel del Corral, J. M.; Castro, M. A.; Gomez-Zurita, M. A. Toxicon 2004, 44, 441-459.

[10]. Rivera, G. Cancer Chemother. Rep. 1975, 59, 743-749.

[11]. Sathisha, A. D.; Hemakumar, K. H.; Basavaraju, Y. B. Ind. J. Heterocycl. Chem. 2007, 17, 15-18.

[12]. Lee, C. T. L.; Lin, V. C. K. Bioorg. Med. Chem. Lett. 1997, 7, 2897-2902.

[13]. Bauer, A. W.; Kirby, W. M.; Sherris, J. C. Am. J. Clin. Pathol. 1966, 39, 493-496.

[14]. Booth, J.; Boyland, E. Biochem. J. 1966, 34, 125-135.

[15]. Basavaraju, Y. B.; Raju, D.; Anupama, N. Int. J. Pharm. Sci. Rev. Res. 2013, 21, 305-309.

[16]. Umesha, B.; Basavaraju, Y. B. Eur. J. Chem. 2013, 4(3), 235-239.

[17]. Sadashivamurthy, B.; Basavaraju, Y. B. Ind. J. Heterocycl. Chem. 2006 , $15,259-262$.

[18]. Furniss, B. S.; Hannaford, A. J.; Smith, P. W. G.; Tatchell, A. R. Vogels text book of practical organic chemistry, $5^{\text {th }}$ edition, ELBS Longman, New York, 1989.

[19]. Lokanatha Rai, K. M.; Murthy, C. A.; Radhakrishna, P. M. Synth. Commun. 1990, 20, 1273-1277.

[20]. Vyas, D. M.; Skonezhy, M. P.; Jenkins, T. A.; Doyle, T. W. Tetrahedron Lett. 1986, 27, 3099-3102.

[21]. Sadashivamurthy, B.; Basavaraju, Y. B. Bulg. Chem. Commun. 2005, 37, 135-139. 